\title{
Isolation of Legionella pneumophila from clinical specimens via amoebae, and the interaction of those and other isolates with amoebae
}

\author{
TIMOTHY J ROWBOTHAM
}

From the Leeds Regional Public Health Laboratory, Bridle Path, York Road, Leeds LS15 $7 T R$

SUMMARY The development of an amoebal enrichment method, for the recovery of viable Legionella pneumophila from clinical materials is described. The method has been used successfully in five of six cases of Legionnaires' disease in which L pneumophila was isolated.

Studies on those L pneumophila isolates led to the discovery that virulent legionellae are attracted to, and attack suitable host amoebae, rather than infection following chance ingestion.

Amoebae are probably natural hosts for Legionella pneumophila and similar bacteria, ${ }^{1}$ therefore it is logical to try and use these protozoa to facilitate the isolation of legionellae from clinical specimens. Amoebae could be used in several ways: (i) to increase the number of legionellae in the specimen; (ii) to protect preferentially legionellae from harmful substances; (iii) to enable isolation of legionellae via micromanipulation. All these strategies have been successfully applied to a sputum sample. Experience suggests that amoebal enrichment followed by acid decontamination and plating on selective media, is a promising method for the isolation of $L$ pneumophila from contaminated clinical material.

Amoebal enrichment requires the exposure of viable legionellae to appropriate viable amoebae in a suitable preparation. Legionellae in clinical specimens may be intracellular, and sometimes aflagellate and therefore cannot seek out amoebae. Because in a primary amoebal enrichment an amoeba has to ingest a viable legionella to become infected, it may be several days before the preparation contains numerous infected amoebae. Subsequent enrichments, particularly liquid enrichments where motile legionellae can seek out the amoebae are much quicker, usually $2-3$ days at $35^{\circ} \mathrm{C}$, a temperature regarded as optimal for the growth of clinical isolates of L pneumophila. To increase the chances of an amoeba coming into contact with a legionella, specimens should be first either liquefied or homogenised, then washed to reduce the concentrations of salt (which can be inhibitory to both

Accepted for publication 24 March 1983 legionellae and amoebae), antibiotics, and other inhibitors. This also reduces the concentration of nutrients, and thus the growth of other microorganisms, (from among which the legionellae eventually have to be isolated), and their products, which could be inhibitory to legionellae or amoebae, of both. As amoebae feed best on surfaces, primar amoebal enrichments are more likely to succees when done in shallow ( $\leqslant 5 \mathrm{~mm}$ ) layers of liquid, of on an agar surface.

\section{Material and methods}

\section{MEDIA FOR AMOEBAE}

Amoeba saline ${ }^{2}$ (Page's modified Neff's amoeba saline). Solution A- $\mathrm{NaCl} 1.20 \mathrm{~g} ; \mathrm{MgSO}_{4} .7 \mathrm{H}_{2} \mathrm{O}$ $0.04 \mathrm{~g} ; \mathrm{Na}_{2} \mathrm{HPO}_{4} 1.42 \mathrm{~g} ; \mathrm{KH}_{2} \mathrm{PO}_{4} 1.36 \mathrm{~g} / 100 \mathrm{ml}$ of glass distilled water. Solution $\mathrm{B}-\mathrm{CaCl}_{2} \cdot 2 \mathrm{H}_{2} \mathrm{O}$ $0.04 \mathrm{~g} / 100 \mathrm{ml}$ of distilled water. Amoeba saline, $10 \mathrm{ml}$ of solution $\mathrm{A}+10 \mathrm{ml}$ of solution $\mathrm{B}+980 \mathrm{ml}$ distilled water. $\mathrm{pH}$ adjusted to $6 \cdot 9^{1}$ with molar $\mathrm{KOH}$.

PYG broth (equivalent to the PYG broth of Drożański $\left.{ }^{3}\right)$ for the axenic cultivation of Acan- $\frac{D}{O}$ thamoeba polyphaga Protoese peptone (Oxoid Ltd Basingstoke, Hants RG24 OPW, UK.) $15 \mathrm{~g}$; yeast N extract powder (Oxoid Ltd) $5 \mathrm{~g}$; D-glucose $10 \mathrm{~g}$; N $\mathrm{FeSO}_{4} 3 \mathrm{mg}$; amoeba saline, one litre. Sterilised by N autoclaving, $115^{\circ} \mathrm{C}$ for $15 \mathrm{~min}$, final $\mathrm{pH}$ adjusted to 6.6.

Amoeba agar (Stoianovitch's malt extract, yeast extract agar ${ }^{2}$ ) Malt extract powder (Oxoid Ltd) $\stackrel{\mathscr{中}}{\rightarrow}$ $0 \cdot 1 \mathrm{~g}$; yeast extract powder $0 \cdot 1 \mathrm{~g}$; agar (Oxoid No 1 ) $10.0 \mathrm{~g} / 1$ of amoeba saline. $\mathrm{pH}$ adjusted to 6.9 with molar KOH. $16 \mathrm{ml} / 90 \mathrm{~mm}$ diameter plate. 
Klebsiella lawns Amoeba agar plates, briefly dried to remove excess moisture, were flooded with a turbid suspension of Klebsiella aerogenes (NCTC 7427) in amoeba saline, drained, and incubated overnight at $35^{\circ} \mathrm{C}$. Before use, the $K$ aerogenes suspension, prepared from growth on a nutrient agar slope, was centrifuged at $650 \mathrm{~g}$ for $10 \mathrm{~min}$ to remove any lumps of agar etc. A thin confluent lawn gave the best results. Thick plates give thicker lawns, which may require a longer exposure to ultraviolet light (UV). Thick plates also allow a greater growth of any contaminants, and may cause problems with microscopy.

UV-killed Klebsiella lawns The above plates were exposed directly under a bactericidal UV lamp for approximately one hour, minimum UV dose $>2 \times 10^{5} \mu \mathrm{Ws} / \mathrm{cm}^{2}$.

UV killed, Klebsiella suspension Growth from one of the above plates was removed with a cotton swab, and resuspended in $5 \mathrm{ml}$ of amoeba saline. The swab was then cultured on half a horse blood agar plate, as a sterility check.

Ditched plates Most plates were divided into two semicircular sections by the removal of a $10 \mathrm{~mm}$ wide strip of agar across the diameter of the plate. Ditching allows the removal of any imperfections in the plate which could harbour survivors on UVirradiated bacterial lawns, and prevents chance contaminants such as fungi from spreading over the whole plate. Antifungal antibiotics usually inhibit the growth of amoebae and therefore cannot be used in amoebal cultures. Some amoebae will grow on legionella media but antifungal antibiotics will prevent this.

\section{MAINTENANCE OF AMOEBAE}

Amoebae were maintained on lawns of UV-killed $K$ aerogenes at room temperature. Both sides of a ditched plate were inoculated with a suspension of trophozoites, or cysts, in amoeba saline, and subcultured from the other end of the plate. Cultures were checked for obvious contaminants by low power microscopy $(\times 100)$ before subculture. Periodic checks for viable bacteria were also made. All amoeba plates were incubated in resealable polythene bags. Sometimes even after the amoebae are subcultured from the part of the plate furthest from the original inoculum, the culture may still not be free from unwanted chance contaminants. Single amoebae or cysts, can as an alternative, be plucked with a micropipette from a plate while it is being examined under a microscope. The single trophozoite isolations in Fig. 1 were done without the aid of a micromanipulator.

When cyst-forming amoebae exhaust their nutrient supply, they encyst. Trophozoite suspensions were prepared from young cultures, cyst suspensions from old cultures. Long term storage of Acanthamoeba spp 6 months, Hartmannella spp 6 months, and Naegleria spp 3 months, was of cyst suspensions in amoebal saline at $4^{\circ} \mathrm{C}$. Vannella platypodia was maintained on plates of live $K$ aerogenes because it grows poorly on UV-killed klebsiellae, and does not form cysts. A polyphaga AP L1501/3A was also maintained axenically in PGY broth at $35^{\circ} \mathrm{C}$, about two divisions occurring each day, 1 to 2 weeks old passages contain few non-viable amoebae.

Some amoebae of the genera Acanthamoeba and Naegleria are pathogenic for man, and therefore amoebae should be treated with respect.

\section{MEDIA FOR LEGIONELLAE}

$B C Y E$ ACES (N-(2-acetamido)-2-aminoethanesulphonic acid) buffered, charcoal (Norit-A Special, $1.5 \mathrm{~g} / \mathrm{l})$, yeast extract, $\alpha$ ketoglutarate medium (BCYE $\left.\alpha^{4}\right)$. $\mathrm{pH}$ adjusted before pouring to $6 \cdot 9 \pm 0 \cdot 02$, at $35-40^{\circ} \mathrm{C}$.

$B M P C=\mathrm{BCYE}+$ cefamandole lithium salt, $4 \mu \mathrm{g}$ (pure)/ml (Eli Lily, Windlesham, Surrey) + polymyxin B sulphate $80 \mathrm{U} / \mathrm{ml}$ (Aerosporin, Burroughs Wellcome, London). ${ }^{4}$ Cycloheximide $80 \mu \mathrm{g} / \mathrm{ml}^{5}$ (as Actidione $85-100 \%$ pure (Upjohn Co, Kalamazoo, Michigan, USA.) replaced anisomycin, ${ }^{4}$ which was unobtainable. Charcoal plates were incubated in air $+2.5 \% \mathrm{vol} / \mathrm{vol} \mathrm{CO}_{2}$, and examined after $3,4,5$ and 10 days. Legionella strains were maintained as suspensions in semisolid agar (Oxoid agar No $11.5 \mathrm{~g} / 1$.) at $4^{\circ} \mathrm{C}$ (Leeds-1A SAP still viable after 14 months). One $3 \mathrm{~mm}$ loopful of growth from a three-day culture on BCYE was added to $5 \mathrm{ml}$ of semisolid agar, and subcultures were made every 3-4 months.

Phosphate buffered saline (PBS) Dulbeco "A" without mineral salts, $\mathrm{pH} 7 \cdot 3$ (Oxoid Ltd).

\section{IMMUNOFLUORESCENCE}

With the exception of formalised BCYE cultures of legionellae, reagents for this work were kindly provided by Dr AG Taylor of the Division of Microbiological Reagents and Quality Control (DMRQC), Central Public Health Laboratory, Colindale, London. Formalin-fixed smears of sputa, hanging drops of amoebal enrichments, and suspect colonies, were stained by an indirect method ${ }^{6}$ with polyvalent $L$ pneumophila (serogroups $1-6$ ) rabbit antisera. (These antisera did not react with formalised BCYE cultures of six other species of the legionella group.) Patients' sera were examined by a similar method, ${ }^{6}$ but with the acetone fixation stage omitted, because in our experience reagent grade acetone slightly reduces the fluorescence. The 


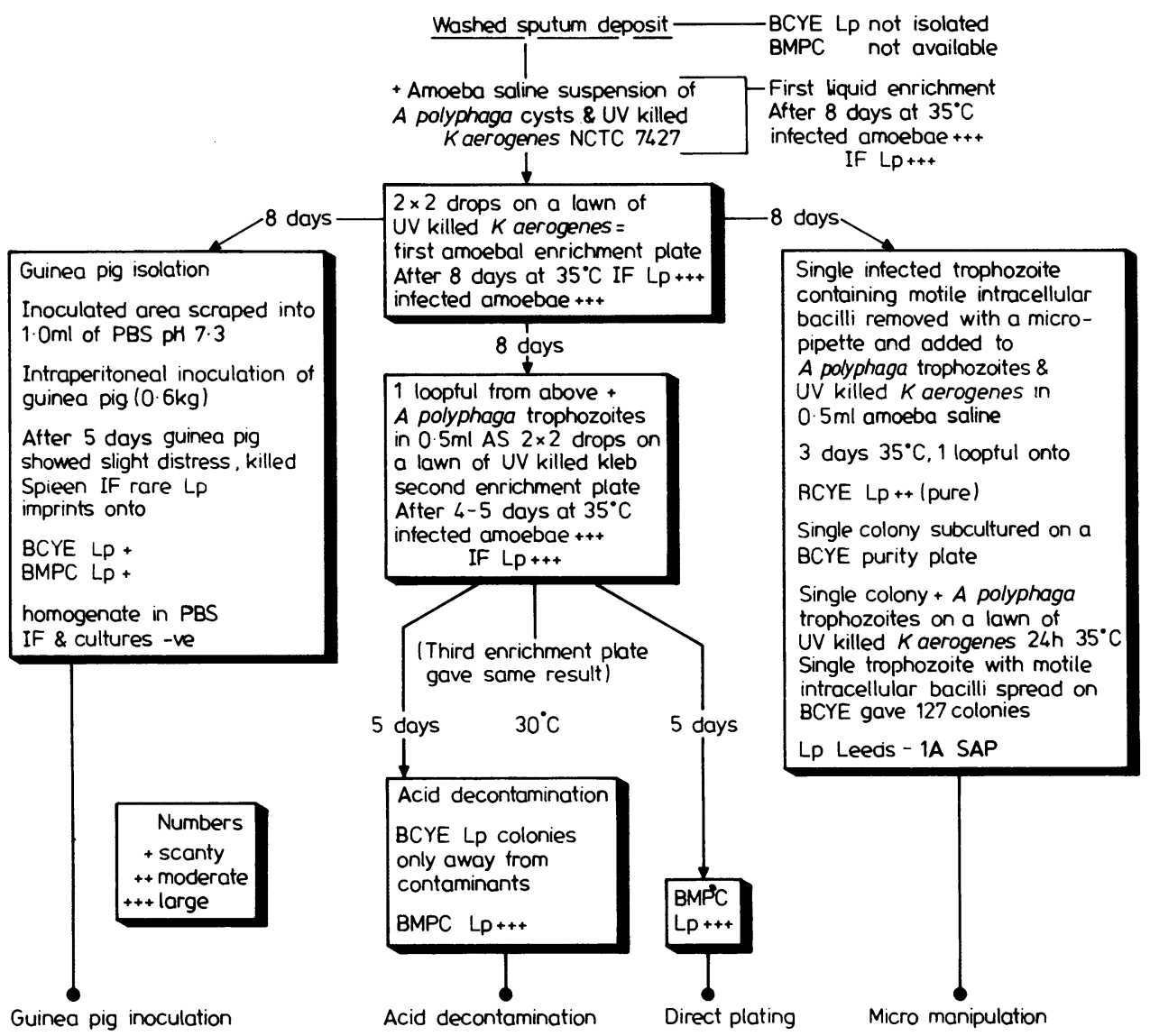

Fig. 1 Isolation of $L$ pneumophila ( $L p)$ from sputum via amoebae (case 1)

mountant used consisted of glycerol, 9 parts; PBS (pH 7.3), 1 part. A positive control serum of known titre, was compared on the same slide as each of the patients sera. Isolates were serogrouped by DMRQC.

\section{GAS LIQUID CHROMATOGRAPHY}

Legionella isolates were also confirmed by analysis ${ }^{7}$ of their fatty acid methyl esters (8, method A). Three-day BCYE cultures were used.

\section{PATHOGENICITY STUDIES}

The 127 colonies of Leeds-1A SAP, obtained when a single infected trophozoite was plated out on BCYE (Fig. 1), were suspended in semisolid agar, stored at $4^{\circ} \mathrm{C}$. Three-day BCYE cultures of this stock, and other isolates, were used to challenge amoebae. The method was the same as that used previously, ${ }^{1}$ in which a wire inoculated with legionellae was drawn across a lawn of UV-killed $K$ aerogenes, in front of, and parallel to, an inoculum of amoebae. The slow growing Acanthamoeba cas tellanii AC L1501/2A and Vannella platypodia $589 / 2$ were inoculated onto the interaction plates several days before the legionella, so that the time taken for the amoebae to reach the legionellae was reduced. Named and numbered strains of amoebae used in these tests were originally obtained from the Culture Centre for Algae and Protozoa (CCAP), 36 Storey's Way, Cambridge CD3 0DT, UK.

\section{AMOEBAL ENRICHMENT}

Acanthamoeba polyphaga (AP L1501/3A) was cho- N sen for enrichment work because it can be infected with $L$ pneumophila (serogroups 1-6) and similar $\tilde{O}$ bacteria, ${ }^{1}$ it does not grow on legionella media, and $\underset{\omega}{N}$ is obtainable as an axenic culture from CCAP. As $L$ pneumophila only attacks feeding trophozoites, $\bullet$ such trophozoites are prefered to cysts for amoebal $\stackrel{\odot}{\Phi}$ enrichment. The relative numbers of amoebae and $\stackrel{\mathscr{C}}{\rightarrow}$ legionellae are important; too few amoebae, and the $T$ amoebae can be destroyed without a marked 


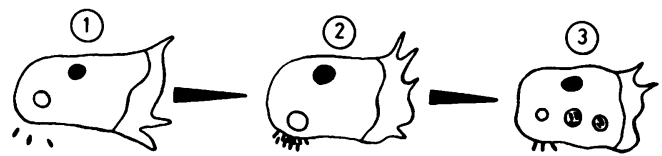

amoebae will excyst and feed. After case 5, the methods for specimen pretreatment and amoebal enrichment were improved, both the original and the improved methods are given below.

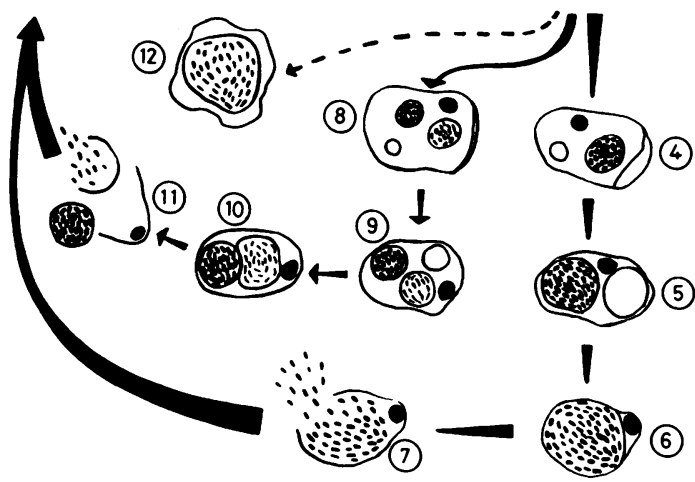

Specimen pretreatment. Sputum. Improved method (The two steps marked* were not in the original methods). After preparation of routine smears and cultures, the remaining sputum was mixed with an equal quantity of Sputasol (Oxoid Ltd) and shaken in a universal bottle on a reciprocal shaker for $15 \mathrm{~min}$. The contents were adjusted to approximately $25 \mathrm{ml}$ with sterile distilled water, mixed, and centrifuged at ${ }^{*}(650 \mathrm{~g}$ for $10 \mathrm{~min}$, to remove cells and debris. The supernatant was then centrifuged at) $2000 \mathrm{~g}$ for $15 \mathrm{~min}$, and the deposit washed* (twice) with distilled water. The washed deposit was used for amoebal enrichment.

Fig. 2 Infection of $\mathrm{A}$ polyphaga with $\mathrm{L}$ pneumophila Leeds-1A SAP (At $30^{\circ} \mathrm{C}$ similar results were obtained for all serogroup 1 isolates described in this paper,

$\mathrm{L}$ pneumophila serogroup $6, \mathrm{~L}$ jordanis, $\mathrm{L}$ bozemanii and L gormanii.

The infective process can be divided into a number of stages, 1) legionellae are attracted to extended trophozoites, 2) they cluster around the rear end of the amoebae, close to where the contractile vacuole empties, 3) the legionellae are phagocytosed, several in each vesicle. 4) The legionellae multiply inside the vesicle, the tightly packed legionellae are non-motile at this stage. 5) The vesicle of legionellae fills most of the cytoplasm, and about this time the contractile vacuole ceases to function properly. 6) A single large vesicle of motile legionellae almost fills the amoeba. 7) The vesicle and amoeba rupture, liberating motile legionellae. 8 \& 9) Sometimes two, rarely three, vesicles of legionellae develop. 10) The legionellae inside them may not become motile at the same time, leading to the release of vesicles of motile or non-motile legionellae, 11) these vesicles may be less than $5 \mu \mathrm{m}(2-8 \mu \mathrm{m}$, mean 4-6 $\mu \mathrm{m})$. It has been postulated that vesicles of legionellae, and infected amoebae are the main infective particles for man (1). Multiple vesicles may occur with agar adapted strains (1). Stage 12). Production of a mature cyst containing motile bacteria, has so far only been accomplished with a legionella-like amoeba pathogen (LLAP-1) and A polyphaga and A palestinensis strains from the same water tank. LLAP-1 does not grow on $B C Y E, B C Y E$ streaked with Staphylococcus aureus (11), or any other media tested so far.

increase in legionellae, too many amoebae, and the amoebae could encyst before the infection spreads to large numbers of amoebae. AP L1501/3A is slower growing than some wild strains, so more trophozoites can be used. A stock suspension of $10^{3}-10^{5} \mathrm{cysts} / \mathrm{ml}$ of amoeba saline has the advantage that it will last about 6 months at $4^{\circ} \mathrm{C}$ and can be screened for viable bacteria before use, but the enrichment conditions must be such that the

Human lung. Original method A small piece of lung $\left(0.1 \mathrm{~cm}^{3}\right)$ was homogenised in sterile distilled water. After allowing for any unwanted coarse material to deposit, the homogenate was centrifuged and washed as above.

Human lung. Improved method Small pieces of lung (total vol $0 \cdot 1 \mathrm{~cm}^{3}$ ) and $2 \mathrm{ml}$ of physiological saline were added to a bijou bottle. The lung was repeatedly squashed with the end of a glass rod, before the contents of the bijou were mixed violently on a vortex mixer for one minute. The resultant "suspension" was made up to approximately $25 \mathrm{ml}$ with sterile physiological saline, mixed, then centrifuged (to remove cells and debris) and washed as above (Improved method).

Amoebal enrichment. Original methods $0.5 \mathrm{ml}$ of an amoeba saline suspension of A polyphaga (AP L1501/3A) cysts, or trophozoites $\left(10^{3}-10^{4} / \mathrm{ml}\right)$ $+0.2 \mathrm{ml}$ of UV-killed $K$ aerogenes suspension, were added to the treated washed specimen. (Cases 1-3 only. Two drops of the above liquid enrichment were placed on each half of a ditched plate of UVkilled $K$ aerogenes, and tilted so that two broad streaks parallel to the ditch were produced.) Secondary liquid enrichments were performed by inoculating one drop or loopful of the previous enrichment into $0.5 \mathrm{ml}$ of an amoeba saline suspension of A polyphaga trophozoites, $+0.5 \mathrm{ml}$ of UV-killed klebsiella suspension.

Amoebal enrichments. Improved methods. Primary enrichments. $1 \mathrm{ml}$ of an amoeba saline washed (three times) suspension of (PYG grown) A polyphaga trophozoites $\left(10^{4}-10^{5} / \mathrm{ml}\right)$ was added to the washed sputum or lung deposits. $0.1 \mathrm{ml}$ of a 
suspension of UV-killed $K$ aerogenes was added to each bottle after two days incubation. Secondary enrichments. Bijoux bottles containing $0.5 \mathrm{ml}$ of an amoeba saline washed suspension of $A$ polyphaga trophozoites $+0 \cdot 1 \mathrm{ml}$ of UV-killed $K$ aerogenes suspension, were inoculated with 1 drop, of the 3 day primary amoebal enrichments.

Amoebal enrichments. Incubation The enrichments were incubated at $35^{\circ} \mathrm{C}$, a temperature regarded as optimal for clinical isolates of $L$ pneumophila, and close to the maximum growth temperature of the $A$ polyphaga strain. Enrichments were examined ${ }^{1}$ frequently for evidence of infected amoebae, and increased numbers of $L$ pneumophila . With the improved methods, examination is required only after three and six days incubation.

Acid decontamination $0.1 \mathrm{ml}$ of an aqueous suspension of material scraped from $1 \mathrm{~cm}^{2}$ of an enrichment plate, or $0.1 \mathrm{ml}$ of a liquid enrichment, $+0.2 \mathrm{~g}$ of tissue grinding ballotini beads and $0.9 \mathrm{ml}$ of $\mathrm{HCl} / \mathrm{KCl} \mathrm{pH} 2.2 \quad(0.2$ molar $\mathrm{HCl}(\mathrm{Hg}$ free) $5.3 \mathrm{ml}, 0.2 \mathrm{M} \mathrm{KCl} 25 \mathrm{ml}$, distilled water to $100 \mathrm{ml}$ (Edelstein ${ }^{4}$ and personal communication) were held at room temperature for $29 \mathrm{~min}$, followed by one minute on a vortex mixer, single drops were plated on BCYE and/or BMPC.

All the methods applied to the first sputum from case 1 , are given in a flowchart, Fig. 1.

\section{Results}

Attempts were made to isolate $L$ pneumophilla by amoebal enrichment from seven cases of Legionnaires' disease. The five cases in which this was successful (numbers 1, 2, 3, 6 and 7, all serogroup 1) are given in detail below. L pneumophila was isolated by direct plating from only three of these five cases, but as most of the specimens were referred from other laboratories they were not examined on the day they were taken and this may have made isolation by direct plating more difficult. In the fourth case (rising titre of 128 to $>512$ to L pneumophila serogroup 1 antigen, DMRQC results) $L$ pneumophila was not isolated from two sputa in which scanty $L$ pneumophila were detected by immunofluorescence, however, the patient had received antibiotics, including erythromycin, for several days before the samples were obtained.

In the fifth case, serogroup 5 L pneumophila was isolated from three mucopurulent sputa from a 68-year-old immunosuppressed leukaemic patient by direct plating on BMPC, but not after liquid amoebal enrichment. The three sputa taken 10, 3, and 3 days before death (no pronounced onset), ${ }_{0}^{0}$ when stained by an indirect fluorescent antibody method for $L$ pneumophila serogroups $1-6$, con- $\frac{\text { o }}{}$ tained numerous moderately bright bacilli with $\mathrm{a}=\overrightarrow{\mathrm{c}}$ morphology consistent with L pneumophila. None was seen in control smears stained with normal rab- $\frac{C}{0}$ bit serum. Two colony types, both serogroup 5 , were $\frac{\bar{c}}{\mathrm{c}}$. isolated from the second sputum sample. The nor- $\vec{\nabla}$ mal type of colony (Leeds-4) consisted mainly of $\varrho$ motile bacilli, whereas the more green type of col- $\infty$ ony (Leeds-4g) consisted of long non-motile bacilli $\vec{\circ}$ and filaments. These colony types were also seen on $-\overrightarrow{-}$ a BCYE isolation plate, and could be subcultured. $\vec{\omega}$ The GLC traces of the two types grown on separate halves of the same plate, were also slightly different.? Two legionellae were detected in one smear of post-mortem lung, but none in a second smear.

$L$ pneumophila was not isolated from post- $\stackrel{\oplus}{-\infty}$ mortem lung, liver or kidney. The patient had ${ }_{\circ}^{\infty}$ received erythromycin for several days before his ${ }^{-}$ death. The serogroup 5 titres obtained by $\mathrm{DMRQC}_{\mathcal{C}}$ for sera taken $46,19,14,10$ and 3 days before the $\mathbb{8}$ patient's death, were $<16,64,64,64$, and 128 需 respectively. This fifth case was the only indigen- $\frac{3}{0}$ ously acquired case.

During the course of this work the methods were $\overrightarrow{-}$ continuously evolving. After the third case liquidoo amoebal enrichment with trophozoites, followed acid decontamination and plating on BMPC used. Further improvements in the methods were incorporated during the investigation of cases 6 ando 7.

\section{CASE 1}

A 69 -year-old woman who smoked 40 cigarettes a day, developed bronchitis, fever, and diarrhoea in November 1981, while on holiday in the Algarve? Portugal. Her symptoms, which also includecक headache, a dry cough, shortness of breath, wheezing, mental confusion, and parathesia, together with? her recent history of foreign travel, suggested Legionnaires' disease. The patient was treated with erythromycin and made an uneventful recovery. She had no $x$-ray evidence of pneumonia. Her antibody? titres to the DMRQC L pneumophila Pontiac-1 serogroup 1 formalised yolk sac antigen ${ }^{9}$ for sera taken $7,10,14$, and 50 days after onset, were $<2 \mathrm{~N}$ 2,16 , and 16 respectively. (DMRQC results $<16 \mathrm{~N}$ $<16$, and 16. 50-day titre provided by the Oxforof Public Health Laboratory.) Similar titres for the 7 ( 10 , and 14 day sera were obtained to formalisedk cultures of Pontiac-1, and the patient's own isolate (Leeds-1A SAP). With the DMRQC formalise $E_{5}$ yolk sac antigen, a fourfold rise in titre to 64 or greater, is regarded as diagnostic for Legionnaires' disease, this mild case did not meet that criterion. $\stackrel{\mathbb{D}}{\mathbb{D}}$ 
A sample of mucopurulent sputum was obtained seven days after onset. In a formalin-fixed smear of the unwashed sputum deposit, stained for $L$ pneumophila (serogroups 1-6) by an indirect immunofluorescence method, 31 brightly fluorescent, short, sometimes slightly curved, aflagellate bacilli, and one cluster of similar but less bright bacilli were seen. None was seen in a control smear treated with normal rabbit serum. A moderately heavy growth of Candida albicans grew on routine culture media inoculated with the liquefied sputum, and on BCYE inoculated with washed sputum deposit. The washing should have removed any inhibitors, nevertheless $L$ pneumophila was not isolated on this medium at this stage. L pneumophila (serogroup 1) was subsequently isolated from the washed sputum deposit by several methods involving amoebae (Fig. 1). Legionellae in the amoebal enrichments were short bacilli, with single subpolar flagellae. All isolates were pathogenic for A polyphaga (Fig. 2). Legionellae were not detected, or isolated from a second mucopurulent sputum taken three days later.

\section{CASE 2}

A 75-year-old man who died suddenly and unexpectedly, apparently of a cold, 10 days after holidaying in Spain and Tangier in February 1982, was found to have a bronchopneumonia at necropsy. A smear of post-mortem lung stained by indirect immunofluoresence for $L$ pneumophila serogroups $1-6$, contained 16 small brightly fluorescent bacilli, none was detected in a control smear stained with normal rabbit serum. A moderate growth of $L$ pneumophila, (Leeds-2) serogroup 1, was isolated by direct plating on BMPC. The post-mortem serum contained no antibody (titre $<8$ ) to $L$ pneumophila serogroups 1-6.

An eight-day liquid amoebal (cyst) enrichment of washed lung deposit contained no amoebae or cysts, scanty L pneumophila were detected by immunofluorescence. After acid decontamination and plating on freshly prepared BMPC, one colony of Lneumophila (Leeds-2A) was isolated. A three-day secondary liquid enrichment contained moderate numbers of $L$ pneumophila by immunofluorescence, and some infected amoebae. In the eight-day primary plate enrichment, scanty to moderate numbers of $L$ pneumophila were detected by immunofluorescence, but $L$ pneumophila was not isolated on BMPC after acid decontamination. No infected amoebae were seen. At first it was thought the poor recovery of $L$ pneumophila from the amoebal enrichments may have been due to the lack of intracellular legionellae, but it was later determined that some of a new batch of agar powder had

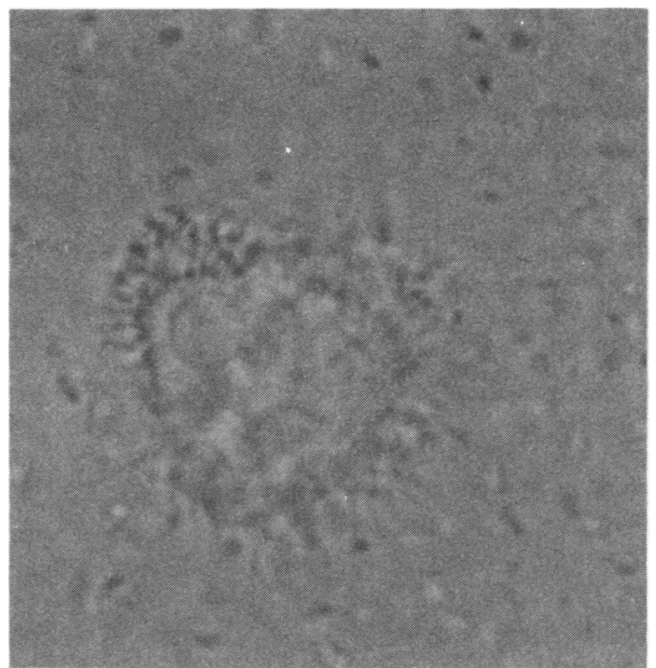

Fig. $3 \mathrm{~L}$ jordanis $(L J)$ clustered around the rear end (top left hand corner of the Figure) of an A polyphaga

(AP L1501/3A) trophozoite. Live material.

been used in the preparation of the BMPC medium, and that this was inhibitory to both legionellae and amoebae. (Oxoid agar No 1 at $10 \mathrm{~g} / \mathrm{l}$, is now used for legionella media.) The L pneumophila isolates obtained by direct plating, and amoebal enrichment were both virulent for $A$ polyphaga (Fig. 2).

\section{CASE 3}

A 58-year-old woman (non-smoker) who had recently returned from a cruise to the Canary Islands, developed fever and pneumonia in May 1982. Her symptoms also included headache, a dry cough, shortness of breath, chest pain, and vomiting. She was treated with erythromycin and penicillin G, and recovered. Her antibody titres to the DMRQC antigen for sera taken $4,7,10$, and 20 days after onset were $<8,16,128$, and 256 respectively. The titres obtained by the DMRQC for these sera were $<16,16,256$, and 256. Four sputa, taken 3, 7, 9, and 10 days after onset, were examined by immunofluorescence for $L$ pneumophila serogroups 1-6. The second sputum contained 10, the third 1, and the fourth 7 , brightly fluorescent coccobacilli/ smear respectively, none was seen in control smears. $L$ pneumophila was not isolated from these three sputa by direct plating on BMPC, or from liquid or plate amoebal enrichments.

The first sputum contained 40 brightly fluorescent coccobacilli and short bacilli, some of the latter were slightly curved; 28 of the bacteria were in one group. Direct cultures on BMPC were negative for $L$ pneumophila. A six-day primary liquid amoebal (trophozoite) enrichment contained no amoebae or 
cysts, scanty to moderate numbers of $L$ pneumophila were detected by immunofluorescence. $0.5 \mathrm{ml}$ of a suspension of A polyphaga trophozoites and UV-killed klebsiellae was added to the enrichment. After $24 \mathrm{~h}$ and acid decontamination, the enrichment was plated out on BMPC, a heavy growth of $L$ pneumophila (Leeds-3A) grew on the plate, except in the inoculum pool, where there were scanty colonies of other bacteria. The immunofluorescence on the seven-day plate enrichment was negative for $L$ pneumophila, and no infected amoebae were seen. After 8 days, the growth on the enrichment plate was removed and suspended in $1 \mathrm{ml}$ of amoeba saline. After five days incubation, large numbers of legionellae were detected by immunofluorescence, and $L$ pneumophila was subsequently isolated after acid decontamination and plating on BMPC. Leeds-3A was virulent for $A$ polyphaga (Fig. 2). The more difficult to prepare plate enrichments were discontinued after this case.

\section{CASE 6}

In early September 1982, a 62-year-old man (nonsmoker) began to complain of severe headache and nausea on the return journey from holiday in Italy. He was admitted to hospital three days later with weakness in the legs and mental confusion, but only minimal respiratory symptoms. Signs of respiratory infection were found at the right base of the lungs, and a diagnosis of a toxic confusional state due to bronchopneumonia and septicaemia was made. $\mathrm{He}$ failed to respond to treatment with flucloxacillin and metronidazole, and died two days after admission. A necropsy revealed lobar pneumonia of the right lower lobe of the lungs, and an enlarged spleen, but nothing else of significance. A plasma sample taken on admission contained no detectable antibody (titre $<8$ ) to L pneumophila serogroups 1-6.

No recognised pathogens were isolated from the lung by conventional techniques. Immunofluorescence for $L$ pneumophila serogroups $1-6$ on three formalin-fixed smears, revealed 8, 9, and 10 brightly fluorescent, small bacilli, consistent with $L$ pneumophila; none was seen on the control smears. Between 35 and 100 colonies of $L$ pneumophila (Leeds-5) (serogroup 1) grew on the direct BMPC plates. Numerous bacteria grew in the amoebal enrichment, and virtually all the amoebae had disappeared within two days. Large numbers of weakly fluorescent bacteria, but no brightly fluorescent legionella-like bacteria were seen. $L$ pneumophila was not isolated from this enrichment. The problem of large numbers of bacteria growing in some amoebal enrichments and inhibiting or destroying the amoebae had occurred before.
The improved specimen pretreatment methods 0 overcame this problem.

In an amoebal enrichment by the improved methods, with $2.4 \times 10^{4}$ PYG broth grown $\overrightarrow{\vec{F}}$ trophozoites, a small increase in the number of $\stackrel{0}{+}$ legionellae was detected after two days, large num- $\frac{\text { ? }}{0}$ bers of legionellae were seen after three days. The $\frac{\bar{\sigma}}{\bar{\omega}}$ first infected amoebae were seen after three days, by $\frac{\hat{\sigma}}{\bar{\phi}}$ six days all the amoebae had been destroyed. After $\varnothing$ acid decontamination, and plating on BMPC, a heavy growth of $L$ pneumophila serogroup 1 was $\vec{\circ}$ isolated from the 3-day (Leeds-5A) and 6-day enrichments. A similar enrichment, containing the $\vec{\omega}$ same number of trophozoites grown on UV-killed $\stackrel{\text { ? }}{\circ}$ klebsiellae and washed free of bacteria, was slightly? slower, with poorer yields of L pneumophila. A heavy growth of $L$ pneumophila was isolated from 0 three-day secondary enrichments inoculated with third day primary enrichments. All isolates were ${ }^{\infty}$ pathogenic for $A$ polyphaga (Fig. 2). Fewer motile legionellae were seen in the enrichments than usual, $\vec{c}$ but at $30^{\circ} \mathrm{C}$ in the pathogenicity studies no differ- $\mathbb{D}$ ences between the strains from this case and the $\frac{\vec{D}}{\mathbb{D}}$ others tested, were noted.

\section{CASE 7}

In December 1982, a 54-year-old woman who h recently returned from holiday in Benidorm, Spain; $\omega$ developed left lower lobe pneumonia. The patifint: was a light smoker (6 cigarettes/day) and a vis moderate drinker; her symptoms included fever shortness of breath, a dry cough, vomiting, confu sion and a toxic urticarial rash; she received $B$ intravenous erythromycin from 10 to 26 days after onset, and made a complete recovery. Her antibody 3 titres to the DMRQC L pneumophila serogroup 1 antigen for sera taken 10,19 and 48 days aftep onset, were $<8,32$ and 32-64 respectively, the DMRQC results for these sera were $<16,32$ and 64. Two small sputum samples taken 10 and 15 days after onset were obtained, these had been stored at $4^{\circ} \mathrm{C}$ for 2.5 days prior to receipt. Rare $L$ pneumophila were detected by immunofluorescence in smears of these sputa, 1 L pneumophila in the first sputum, and 11 in the second, none was seen in control smears. The first sputum was storedE. for an additional six days at $-20^{\circ} \mathrm{C}$ before amoebaN enrichment. The amoebal enrichments (by the्r improved methods) initially contained $2.6 \times 100$ amoebal saline washed, broth grown, $A$ polyphaga trophozoites. Three small colonies of L pneumophila (Leeds-6, serogroup 1) grew on the direct BMPC plate inoculated with the first sputum o but these were soon overgrown by $C$ albicans. In the amoebal enrichment of the first sputum, most of the amoebae had been destroyed after three days, anfक्ष 
numerous small $L$ pneumophila were detected by immunofluorescence. After acid decontamination, and plating on BMPC, a heavy growth of $L$ pneumophila was isolated from the three-day (Leeds-6A, serogroup 1) and six-day enrichments, no growth of legionellae occurred in the inoculum pools on these plates, where scanty colonies of $C \mathrm{al}$ bicans were present. The inhibition by $C$ albicans was very marked on the three-day enrichment plate, which because of an incubator fault, had been incubated at room temperature for most of the first three days, thus allowing the candida to grow on the plate before good conditions for the growth of $L$ pneumophila were established. L pneumophila was not isolated from the second sputum by any method. Leeds-6 \& 6A, were both virulent for A polyphaga (Fig.2).

\section{PATHOGENICITY STUDIES}

All the serogroup 1 isolates in this study were virulent for $A$ polyphaga AP L1501/3A at $30^{\circ} \mathrm{C}$ (Fig. 2). When Leeds-1A SAP was inoculated directly into the advancing edge of a colony of $A$ polyphaga on a lawn of UV-killed klebsiellae, the first rounded-up amoebae containing motile legionellae were seen in just under $24 \mathrm{~h}$ at $35^{\circ} \mathrm{C}$, one and a half days at $30^{\circ} \mathrm{C}$, and three days at room temperature. A similar result was obtained when lawns of live $K$ aerogenes .were used. Thus $K$ aerogenes, which is markedly inhibitory to $L$ pneumophila on BCYE and BMPC media, apparently does not inhibit the intracellular growth of $L$ pneumophila. At $30^{\circ} \mathrm{C}$ Leeds-1A SAP also infected $A$ castellanii AC L1501/2A, a rapid growing Acanthamoeba strain of the polyphaga group from a local humidifier, ${ }^{1}$ and three strains of $A$ polyphaga and one of $A$ palestinensis from a local drinking water tank. When nearly all the amoebae had been destroyed on the interaction plates, the material in the vicinity of the line contained numerous small motile legionellae. Within $20-40 \mathrm{~min}$ of adding a few feeding uninfected trophozoites to the hanging drops, these legionellae were attracted to the trophozoites, and clustered at a point towards the rear of the amoeba where the contractile vesicle emptied. Attraction to rounded-up trophozoites was much less, or absent, and was not seen towards obviously infected trophozoites, or cysts. The attracted legionellae were subsequently phagocytosed, several per vesicle. This behaviour was also observed after trophozoites were added to the primary amoebal liquid enrichment of case 3 , and has now also been seen between $A$ polyphaga and several other members of the legionella group-for example, L pneumophila serogroup 6 (RSW SC-1, \& RSW PR-3), L jordanis BL-540 (Fig. 3), $L$ bozemanii (WIGA, NCTC 11368) \& L gormanii
(LS-13). Attenuated strains of $L$ pneumophila are usually non-motile and thus do not show it. A strain of Alkaligenes $s p$, isolated from the primary liquid amoebal enrichment of the first sputum sample from case 4, was also attracted to, and clustered around A polyphaga trophozoites, but was apparently not ingested, nor did they infect the amoebae. Bacteria showing this type of behaviour might obstruct the infection of amoebae by legionellae. Leeds-1A SAP could also infect Naegleria gruberi 1518/1E, $N$ jadini B 1518/2, N lovaniensis (recent isolate from an industrial hot water spray) and Hartmannella vermiformis B 1534/7A, however infected amoebae were much rarer and required a heavier inoculum of legionellae to be produced. Leeds 1A SAP from these amoebae and from A polyphaga (AP L1501/ $3 \mathrm{~A}$ were, not attracted to, nor did they cluster around these amoebae; and were actually seen to shy away from $N$ gruberi $1518 / 1 \mathrm{E}$. It therefore appears that legionellae can actively seek out, and attack suitable hosts, perhaps avoiding others. Leeds-1A SAP was not attracted to, and did not infect Vannella platypodia 1589/2.

The serogroup 5 isolates of $L$ pneumophila from the fifth case, were not attracted to, nor did they cluster around A polyphaga AP L1501/3A trophozoites. A heavy inoculum of these legionellae was required to produce infected amoebae, thus explaining the failure of amoebal enrichment in this case. As different serogroups of $L$ pneumophila apparently have different host specificitier, there is the possibility that amoebal enrichments could be made selective for certain groups of legionellae.

\section{Discussion}

Sputasol contains dithiothreitol which can be inhibitory to $L$ pneumophila.$^{10}$ Sputasol however is unlikely to have affected the recovery of $L$ pneumophila from case 1 , because when tenfold dilutions of a suspension of Leeds-1A SAP in distilled water were incubated with equal volumes of either Sputasol, physiological saline, or distilled water, for $30 \mathrm{~min}$ at $37^{\circ} \mathrm{C}$, no decrease in colony size, or recovery, on BMPC plates was noted with the sputasol treated suspensions (AD Muirhead, Leeds PHL, personal communication).

$C$ albicans isolated from the first sputum of case 1 , streaked across a lawn of $L$ pneumophila (Leeds1A SAP) on BCYE, greatly reduced the growth of legionellae in the vicinity of the streak. In case 5 no $L$ pneumophila colonies were seen near $C$ albicans colonies on BCYE and BMPC plates inoculated with sputum, and in case 7 inhibition of $L$ pneumophila by $C$ albicans was again observed. Thus in case 1 , the heavy growth of $C$ albicans on 
the BCYE isolation plate inoculated with washed sputum deposit, may have prevented the direct isolation of $L$ pneumophila. Growth of $C$ albicans on BCYE can be prevented by adding anisomycin, ${ }^{4}$ which is now obtainable, but very expensive.

Liquid amoebal enrichments of environmental specimens can suffer from predation by other organisms, but this is unlikely to occur with clinical specimens. Compared with an agar plate enrichment, a liquid enrichment can be prepared at any time from refrigerated suspensions, broth or plate cultures, therefore it was liquid amoebal enrichments which were selected for development. Cases 6 and 7 demonstrate the improvements in techniques during the past year. Although so far only a few positive specimens have been examined by the improved enrichment methods, the enrichments, all for $L$ pneumophila serogroup 1, have worked well after three days. Secondary enrichments for most specimens will probably be unnecessary.

\section{Conclusions}

$L$ pneumophila can be isolated from suitable amoebal enrichments of clinical specimens. Liquid amoebal enrichments are easy to prepare with axenic broth cultures of amoebae. Amoebal enrichment has also led to a better understanding of the interactions between legionellae and their natural hosts.

The author would like to thank Eli Lily \& Co for the cefamandole, Dr RJ Fallon (Ruchill Hospital, Glasgow) for the $L$ jordanis \& $L$ gormanii strains, medical colleagues for permission to publish details of their patients, and Dr GL Gibson for his encour agement and constructive criticism during the preparation of this paper.

\section{References}

' Rowbotham TJ. Preliminary report on the pathogenicity o Legionella pneumophila for freshwater and soil amoebae. Clin Pathol 1980;33:1179-83.

${ }_{2}^{2}$ Page FC. An illustrated key to freshwater and soil amoebae with notes on cultivation and ecology. Freshwater Biologica Association, Ambleside, Cumbria, England. Scientific Publies cation No 34, 1976.

${ }^{3}$ Drozianski W. The in vitro experiments on the fatal infection of Amoebida (Kent, 1880) by pathogenic bacterium. Acta Pro $-\overrightarrow{-}$ tozol Polon 1972;10:275-86.

${ }^{4}$ Edelstein PH. Improved semiselective medium for isolation of Legionella pneumophila from contaminated clinical anf environmental specimens. J Clin Microbiol 1981;14:298-303w

${ }^{5}$ Bopp CA, Sumner JW, Morris GK, Wells JG. Isolation of Legionella spp from environmental water samples by low-ph treatment and use of a selective medium. J Clin Microbio 1981;13:714-9.

' Fallon RJ. Laboratory diagnosis of Legionnaires' disease $A C P$ Broadsheet 99, 1981.

${ }^{7}$ Moss CW, Weaver RE, Dees SB, Cherry WB. Cellular fatty acid composition of isolates from Legionnaires' disease. J Clind Microbiol 1977;6:140-3.

- Tisdall PA, Roberts GD, Anhalt JP. Identification of clinica isolates of mycobacteria with gas-liquid chromatograph alone. J Clin Microbiol 1979;10:506-14.

' Harrison TG, Taylor AG. Diagnosis of Legionella pneumophilainfections by means of formalised yolk sac antigens. $J$ girbo Pathol 1982;35:211-4.

${ }^{10}$ Pine L, George JR, Reeves MW, Harrell WK. Development clinically defined liquid medium for growth of Legiof êllo pneumophila. J Clin Microbiol 1979;9:615-26.

"Hopfer RL, Mills K, Fainstein V, Fischer HE, Luna MP Pneumonia caused by a previously undescribed bacterium. Clin Microbiol 1982;16:536-41.

Requests for reprints to: Dr TJ Rowbotham, Public Health Laboratory, Bridle Path, York Road, Leeds LS15 7TR? England. 